\title{
Improved data acquisition efficiency for respiratory motion correction in coronary MRI
}

\author{
Mehdi H Moghari ${ }^{1,2^{*}}$, Sébastien Roujol ${ }^{1,2}$, Markus Henningsson ${ }^{1,2}$, Raymond H Chan ${ }^{1,2}$, Susie Hong ${ }^{1,2}$, \\ Beth Goddu ${ }^{1,2}$, Lois A Goepfert ${ }^{1,2}$, Kraig V Kissinger ${ }^{1,2}$, Warren J Manning ${ }^{1,2}$, Reza Nezafat ${ }^{1,2}$ \\ From 15th Annual SCMR Scientific Sessions \\ Orlando, FL, USA. 2-5 February 2012
}

\section{Summary}

To investigate the performance of a novel algorithm for correcting respiratory-induced heart motion for wholeheart coronary MRI.

\section{Background}

A right hemi-diaphragm (RHD) respiratory navigator is commonly used to suppress the respiratory motion of the heart in coronary MRI [1]. Typically, a small $5 \mathrm{~mm}$ end expiratory gating window $(\mathrm{GW})$ is used to gate data. While this technique successfully suppresses respiratory motion, it prolongs scan time due to navigator efficacies of only $30-50 \%$. Increasing the GW to $15 \mathrm{~mm}$ would increase the navigator efficiency. In this study, we present a novel respiratory motion correction algorithm which allows increasing the GW to $15 \mathrm{~mm}$.

\section{Methods}

Whole-heart coronary MRI with isotropic $1.3 \mathrm{~mm}^{3}$ resolution was acquired using partial Fourier and a navigator with $15 \mathrm{~mm}$ GW on a $1.5 \mathrm{~T}$ Philips CMR scanner from 10 healthy subjects ( 4 males; $29 \pm 13 \mathrm{yr}$ ). The navigator RHD positions were used to sort the acquired k-space segments into 15 separate $1 \mathrm{~mm}$ bins (based on their displacement). To compensate for the respiratory motion of the heart, all $\mathrm{k}$-space segments acquired in each bin were assigned a 3D translation parameter. The 3D translation parameter was estimated using an iterative gradient descent optimization algorithm to correct the kspace segments such that the sharpness of the image, reconstructed using the corrected k-space segments, is maximized. The variance of the gradient of the image was used as the measure for the image sharpness [2].

${ }^{1}$ Harvard Medical School, Cambridge, MA, USA

Full list of author information is available at the end of the article
For comparison, another whole-heart coronary MRI dataset was acquired using the same sequence and a navigator with a $5 \mathrm{~mm} \mathrm{GW}$.

\section{Results}

Fig.1 displays multi-planner reformatted images acquired using a $5 \mathrm{~mm}$ GW (A), a $15 \mathrm{~mm}$ GW without correction (B) and a $15 \mathrm{~mm}$ GW with the proposed correction technique $(C)$. There is an improvement between images acquired using $15 \mathrm{~mm}$ GW with correction. The mean and standard deviation of scan acquisition time and efficiency as well as mean and standard deviation of normalized vessel sharpness of right coronary artery (RCA), left anterior descending (LAD) and left circumflex (LCX) arteries are shown in Table 1. However there is an improvement in the mean of the sharpness of LAD and LCX using the proposed technique compared to the reference, but it is not significantly different.

\section{Conclusions}

We present a novel retrospective RHD motion compensation algorithm for whole-heart coronary MRI that allows for widening GW and reduces scan acquisition time by a factor of 2 .

\section{Funding}

NIH.

\section{Author details \\ ${ }^{1}$ Harvard Medical School, Cambridge, MA, USA. ${ }^{2}$ Medicine, Beth Isreal Deaconess Medical Center, Boston, MA, USA.}

Published: 1 February 2012

\section{References}

1. Ehman : Radiology. 1996.

2. Pacheco : Pattern-Recognition. 2000. 


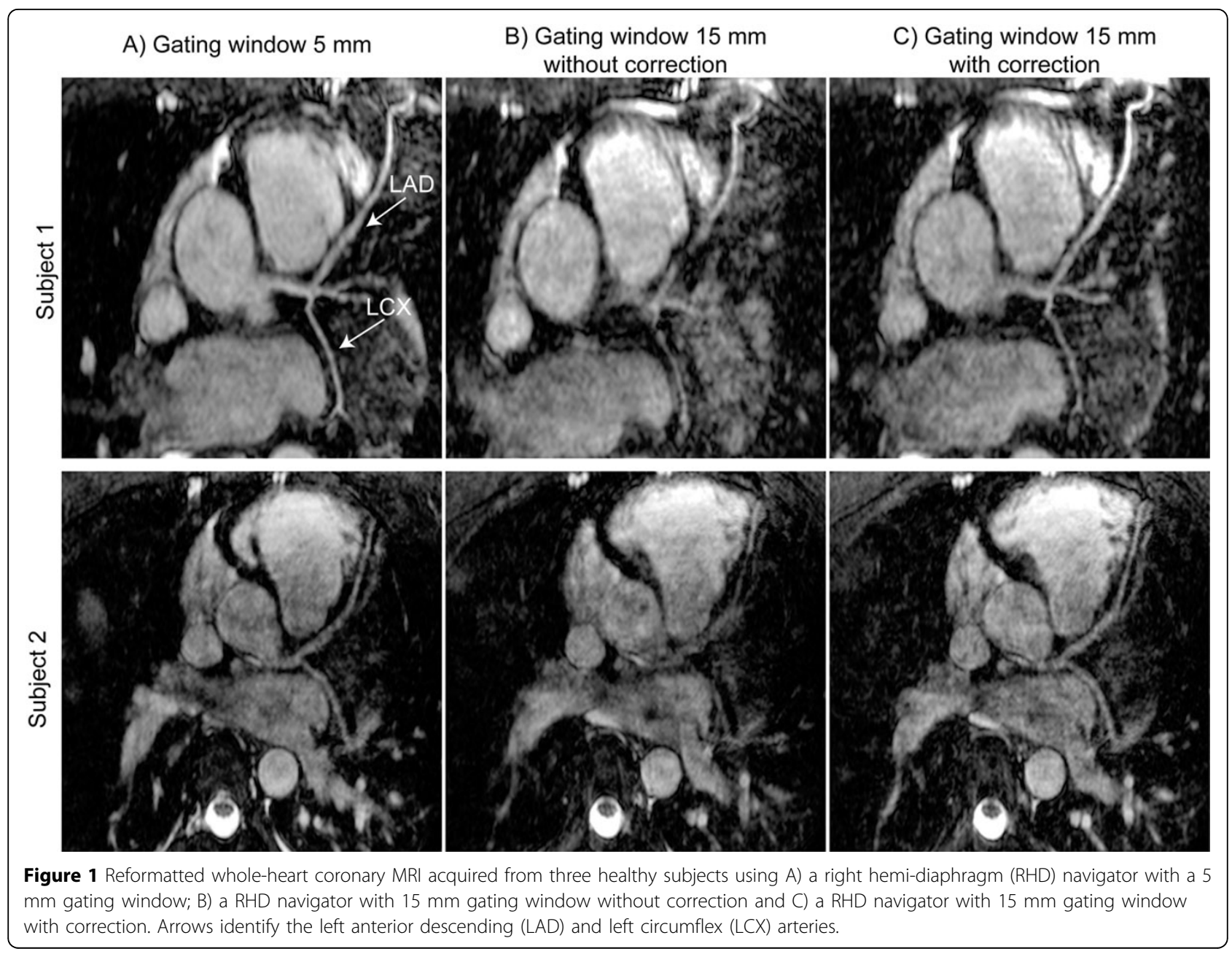

Table 1 Quantitative comparison between the whole-heart coronary MRI acquired with a navigator with a $5 \mathrm{~mm}$ gating window (GW), $15 \mathrm{~mm} \mathrm{GW}$ without correction (noMC), and $15 \mathrm{~mm}$ GW with correction (MC) using the proposed algorithm. Higher values of sharpness are superior. All values are reported as mean \pm standard deviation, and all statistically significant $\boldsymbol{p}$ values are in bold.

\begin{tabular}{|c|c|c|c|c|c|c|}
\hline \multirow[t]{2}{*}{ Parameter } & \multirow{2}{*}{$\begin{array}{l}5 \mathrm{~mm} \text { GW } \\
\text { (REF) }\end{array}$} & \multirow{2}{*}{$\begin{array}{l}\text { 15mm GW without Correction } \\
\text { (noMC) }\end{array}$} & \multirow{2}{*}{$\begin{array}{l}15 \mathrm{~mm} \text { GW with Correction } \\
(\mathrm{MC})\end{array}$} & \multicolumn{3}{|c|}{$p$ value $(n=10)$} \\
\hline & & & & MC vs. REF & noMC vs. REF & MC vs. noMC \\
\hline $\begin{array}{l}\text { Imaging time } \\
\text { (min.) }\end{array}$ & $14 \pm 5$ & $7 \pm 1$ & $7 \pm 1$ & $<0.002$ & $<0.002$ & - \\
\hline Scan efficiency (\%) & $55 \pm 13$ & $92 \pm 8$ & $92 \pm 8$ & $<0.001$ & $<0.001$ & - \\
\hline $\begin{array}{l}\text { RCA sharpness } \\
\left(\mathrm{mm}^{-1}\right)\end{array}$ & $0.51 \pm 0.10$ & $0.42 \pm 0.17$ & $0.49 \pm 0.13$ & 0.692 & 0.151 & 0.298 \\
\hline $\begin{array}{l}\text { LAD sharpness } \\
\left(\mathrm{mm}^{-1}\right)\end{array}$ & $0.37 \pm 0.15$ & $0.29 \pm 0.16$ & $0.39 \pm 0.04$ & 0.683 & 0.271 & 0.079 \\
\hline $\begin{array}{l}\text { LCX sharpness } \\
\qquad\left(\mathrm{mm}^{-1}\right)\end{array}$ & $0.33 \pm 0.25$ & $0.21 \pm 0.19$ & $0.38 \pm 0.15$ & 0.574 & 0.271 & $<0.047$ \\
\hline
\end{tabular}

